\title{
L'OSSERVATORIO GEOFISICO INSTALLATO \\ A KHARTOUM IN OCCASIONE DELL'ECLISSE DI SOLE DEL 25-2-1952
}

\author{
R. Cialdea - P. Dominici
}

In occasione dell'eclisse totale di sole del 25 febbraio 1952 l'Istituto Nazionale di Geofisica ci incaricò di impiantare un Osservatorio temporaneo a Kbartoum (Sudan) per rilevare, durante l'eclisse, le eventuali variazioni delle proprietà elettriche dell'atmosfera, del campo magnetico terrestre, dello stato di polarizzazione della luce diffusa dell'atmosfera e della temperatura virtuale del cielo.

Nella presente relazione riferiremo sull'impianto di questo Osservatorio e sul suo funzionamento.

Il materiale scientifico, di cui alla precedente relazione $\left({ }^{1}\right.$, era stato già tutto preparato nella sede centrale di Roma dell'I.N.G. e pronto per la spedizione. Le apparecchiature scientifiche ed il materiale vario necessario occupavano complessivamente nove casse, per un peso di $635 \mathrm{~kg}$. A causa delle particolari situazioni politiche dell'Egitto e del Sudan, si ritenne opportuno, per poler sorvegliare personalmente il prezioso materiale affidatoci, di compiere il viaggio per mare fino ad Alessandria e quindi via terra fino a Khartoum. Fu stabilito di lasciare l'Italia il 27 gennaio con la $\mathbf{M} / \mathbf{n}$ Esperia, ciò che faceva prevedere l'arrivo a Khartoum per il 7 febbraio. In tal modo si avevano a disposizione ben diciotto giorni prima dell'eclisse per una completa messa a punto di tutti gli apparecchi. Le casse contenenti la stazione magnetica Ruska ci sarebbero state inviate dopo per via aerea, data l'estrema delicatezza dei suoi strumenti.

In seguito ad accordi intercorsi fra l'Istituto Nazionale di Geofisica e l'Osservatorio Astronomico di Roma su Monte Mario, fu stabilito di effettuare il viaggio di andata insieme al prof. Massimo Cimino incaricato da detto Osservatorio per studi di carattere astronomico. Anche le pratiche concernenti i contatti con il Governo Sudanese e le questioni connesse con il viaggio, come $i$ passaporti, $i$ visti ed $i$

(1) R. Cialdea: Ann. di Geofisica, vol. V, n. 3, 1952. 
lasciapassare delle diverse dogane furono svolte in comune con il prof. Cimino. Dato il carattere scientifico della spedizione, tutte le pratiche relative alle varie dogane furono facilmente espletate, ottenendo l'esportazione temporanea dall'Italia, il libero transito in franchigia doganale ed uno speciale "agreement ) col Governo Sudanese per l'entrata e l'uscita del nostro materiale nel Sudan senza gravami fiscali.

Partiti da Napoli il 27 gennaio giungemmo ad Alessandria il 30. $111^{\circ}$ febluraio partimmo dal Cairo con il treno espresso in coincidenza con la linea bisettimanale Shellal-Wadi Halfa-Khartoum. Purtroppo un incidente occorso al treno nel tratto Cairo-Shellal ci fece ritardare di tre giorni l'arrivo a Khartoum, dove giungemmo il 10 febbraio.

L'11 febbraio fu impiegato nella ricerca di una localita adatta all'impianto dell'Osservatorio. Le condizioni alle quali doveva soddisfare questa località erano: 1) essere situata in un luogo il più possibile aperto, senza notevoli limitazioni all'orizzonte visibile; 2) essere situata in una zona non affetta da disturbi elettrici e magnetici; 3 ) offrire sufficienti garanzie, per la sicurezza e la conservazione in efficienza degli apparecchi. Il Governo Sudanese aveva messo a disposizione delle varie spedizioni alcune aree, raggruppate in due punti: il primo (Northern Barracks) in prossimità del ponte ferroviario sul Nilo in una zona molto disturbata per la presenza di linee tranviarie; il secondo (Kilo-five) in pieno deserto e quindi da scartarsi per $i$ danni che la sabbia avrebbe potuto causare ad apparati delicatissimi quali erano i nostri. Infatti la zona di Khartoum era sempre soggetta ad un debole vento del nord $(f<3)$, sufficiente a riempire di una sabbia finissima qualunque ambiente cbe non fosse a tenuta ermetica.

Fortunatamente ottenemmo la preziosa collaborazione della Missione Cattolica che mise a nostra disposizione la scuola a $\mathrm{S}$. Francesco Saverio ). Questa scuola si trovava alla periferia meridionale della città, in una zona non molto disturbata né elettricamente né magneticamente. Essa era dotata di un grande cortile cintato (circa $70 \times 70$ metri), dal centro del quale l'altezza angolare degli ostacoli circostanti era inferiore ai $10^{\circ}$. Si aveva inoltre il vantaggio che il vento prerlominante del Nord, passando attraverso la città, depositava quasi tutta la sabbia di cui poteva esser carico; né la città, composta quasi esclusivamente di ville con giardini e parchi poteva perturbare l'Osservatorio dal punto di vista elettrico. Solo alcuni rari treni a vapore portavano dei brevi disturbi, d'altronde ben individuabili sulle registrazioni elettriche. Dall'altro lato della scuola, il lato Sud, c'era il deserto; 
il vento spirava da questo lato solo eccezionalmente, ma con grande violenza, provocando delle vere e proprie tempeste di sabbia. Fortunatamente questa circostanza si è verificata solo due volte durante il periodo di funzionamento dell'Osservatorio. La scuola «S. Francesco Saverio " soddisfaceva alle necessarie condizioni, e fu senz'altro scelta per l'impianto 'dell'Osservatorio.

Il 12 febbraio iniziammo il montaggio degli apparati, che procedette con grande rapidità, tanto più che avevamo ottenuto anche l'uso di una grande rimessa che attrezzammo a laboratorio. Un'altra piccola stanza, concessaci in seguito, fu adibita a camera oscura.

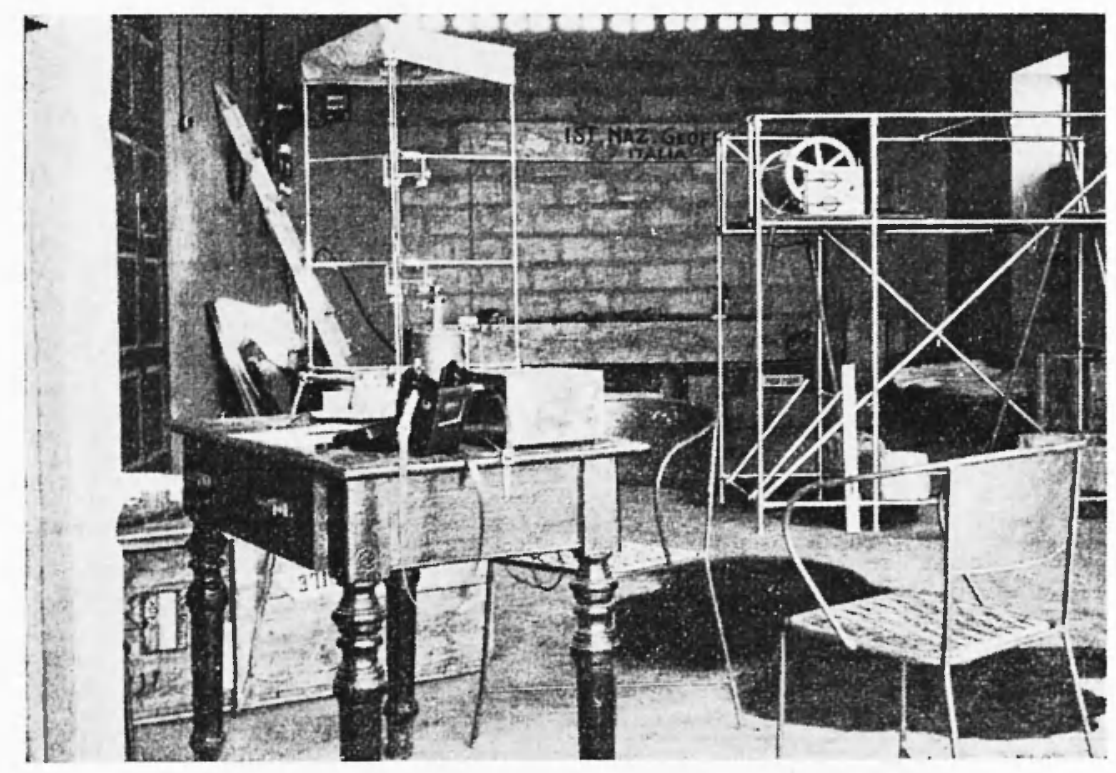

Fig. 1

Nell'interno della rimessa sistemammo l'officina da campo, le batterie di accumulatori per $i$ vari servizi elettrici, il registratore e la stazione magnetica. La fig. 1 mostra l'interno di questo lahoratorio durante la fase di montaggio degli apparati; a destra si nota l'intelaiatura del registratore, ed a sinistra quella del nefoscopio fotografico.

La planimetria di fig. 2 rappresenta le due ali della scuola e la rimessa: vi è anche indicata la direzione del Nord geografico. I vari simboli contraddistinti con le lettere $a, b$, ecc. indicano la posizione degli apparati usati per le varie misure, e che ora descriveremo brevemente. 
Temperatura virtuale del cielo. - L'apparato di Gorczynski usato per questa misura è costituito da una serie di coppie termoelettriche, le cui saldature dispari, annerite, sono disposte in modo da formare una superficie orizzontale di circa $1 \mathrm{~cm}^{2}$ che, ricoperta da una calotta di vetro, viene esposta in luogo aperto. Questo apparato, contrassegnato nella fig. 2 con la lettera $g$, venne disposto sulla terrazza più alta della scuola, cioè quella della rimessa, da dove praticamente era visibile tutto l'orizzonte. Le misure venivano eseguite saltuariamente durante

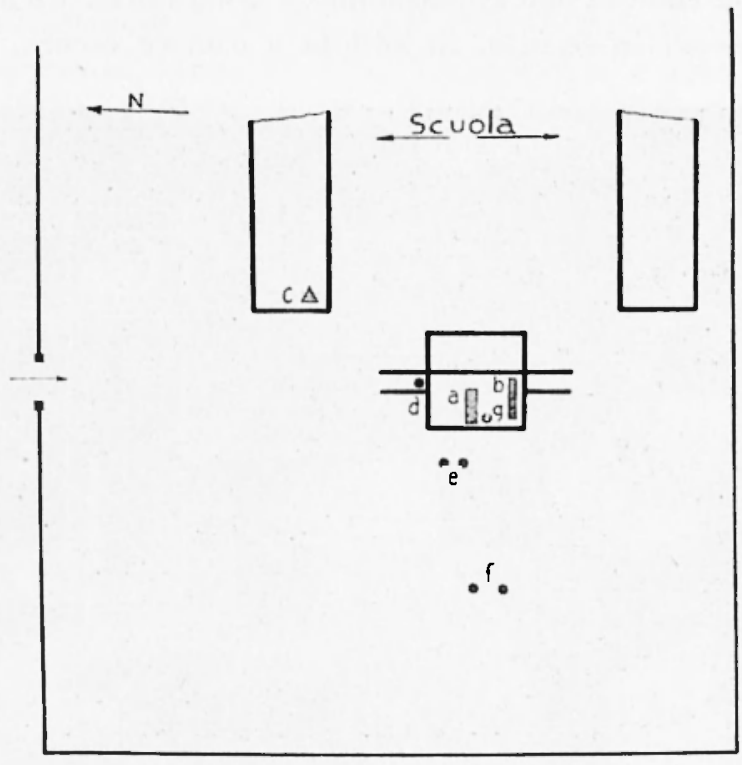

Fig. 2

la giornata, spesso al tramonto, escludendo di volta in volta con adatti schermi la radiazione diretta del sole.

Nefoscopio fotoelettrico. - In una nota precedente $\left({ }^{2}\right)$ erano state date le norme per la sistemazione di tale' apparato, in modo che l'influenza dovuta alla diversa posizione del sole sulla volta celeste durante la giornata fosse ridotta al minimo; per annullare questa variazione sistematica diurna $\grave{e}$ in pratica sufficiente dirigere l'apparato verso il polo celeste. Purtroppo l'altezza del polo sull'orizzonte a Khartoum era molto bassa (circa $15^{\circ}$ ), di guisa che, se si fosse puntato

(2) R. Cialdea: Ricerca scient. e Ricostruzione 17 (1947), p. 633. 
l'apparato verso il polo, si sarebhe registrata esclusivamente la luce diffusa dalle bassissime regioni dell'atmosfera, e quindi il grado di polarizzazione si sareble mantenuto sempre molto basso indipendentemente dalla torbidità dell'atmosfera. Per conciliare la necessità di eliminare la variazione diurna con quella di poter misurare gradi di

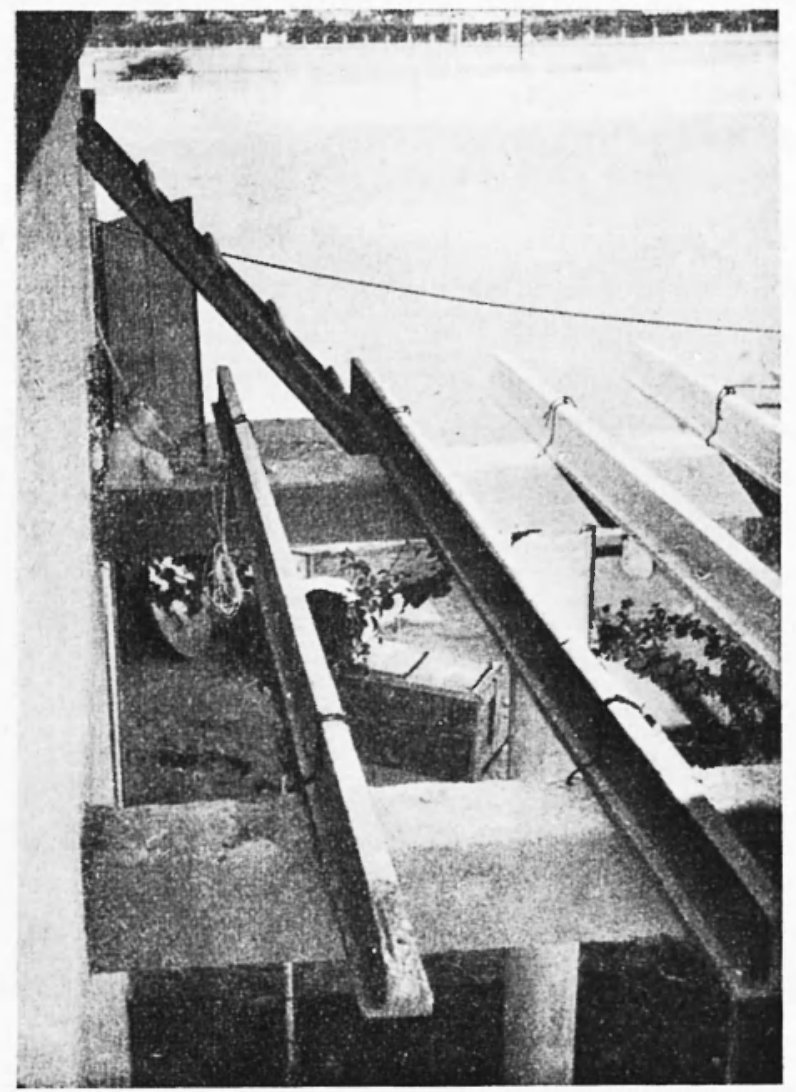

Fig. 3

polarizzazione abhastanza forti da essere indicativi nei riguardi della torbidità dell'atmosfera, si è diretto l'apparato verso una zona a $20^{\circ}$ dal polo celeste, cioè ad una distanza zenitale di $55^{\circ}$, sempre nel piano del meridiano locale. La variazione diurna risultante è stata poi calcolata con i metodi accennati nella nota citata. L'apparato fu sistemato nella parete Nord della rimessa ed è contraddistinto nella planimetria con un circoletto nero e la lettera $d$; è visibile in dettaglio nella fig. 3 , che rappresenta la parete Nord della rimessa ed una parte del cortile. 
Nefoscopio fotografico. - Anche questo apparato aveva bisogno del massimo orizzonte libero, e pertanto andava sistemato su una terrazza. Si usufrui della terrazza di una delle ali della scuola, in modo che l'operatore che doveva azionare le macchine fotografiche non perturbasse le altre misure di radiazione che venivano effettuate sulla terrazza della rimessa. Nella planimetria il nefoscopio fotografico $\dot{e}$ indicato con un triangolino e la lettera $c$. L'apparato è visibile nella fig. 1 della nota precedente $\left({ }^{1}\right)$. I rilievi fotografici erano seguiti saltuariamente durante il giorno.

Elettricità atmosferica. - Le sonde di potenziale del tipo radioattivo a polonio, preparate da noi stessi prima della partenza, erano sistemate alla sommità di due stili metallici alti rispettivamente 1 metro e 1,5 metri. Ciascuno stilo era isolato alla base da un grosso blocco di elettrina. Un sottile filo di rame collegava le sonde agli elettrometri del registratore, attraversando una finestra della rimessa con un isolatore passante in elettrina. Non vi era alcun isolatore di sostegno fra le sonde e la finestra, dal momento cbe il peso del filo era pressoché trascurabile, e gli stili erano saldamente trattenuti da una grossa base di ghisa. $\mathrm{Si}$ aveva così il vantaggio di eliminare un certo numero di isolatori liberi in aria: avevamo infatti osservato che, a causa della extrema secchezza dell'aria e della finissima sabbia trasportata talvolta dal vento, gli isolatori si caricavano spesso per semplice azione del vento. Questo ci costrinse a proteggere accuratamente dal vento tutti gli isolatori sia delle sonde cbe dei dispersori per la misura della conducibilità positiva e negativa.

Le due sonde erano poste a circa sette metri una dall'alira, in modo da non influenzarsi reciprocamente a causa della deformazione delle superfici equipotenziali prodotta dagli stili metallici. I dispersori furono disposti con analoghi accorgimenti, ma più vicino alla rimessa. Le quattro linee ad isolamento elettrostatico pervenivano ai rispettivi elettrometri situati nell'interno del registratore. I dispersori, di forma sferica, erano automaticamente caricati ogni minuto primo a circa 100 volt positivi per l'uno, negativi per l'altro. A ciò provvedeva un relais elettrico comandato da un orologio che, a mezzo di contatti isolati in polistirene, connetteva $\mathrm{i}$ dispersori a due batterie a secco. Due o tre volte al giorno veniva eseguito l'azzeramento degli elettrometri e la loro taratura a mezzo di un elettrometro a lettura diretta Triil, 
Täuber. Nella planimetria i circoletti $e$ ed $f$ rappresentano rispettivamente i dispersori e le sonde.

Stazione magnetica Ruska. - Questa stazione fu sistemata nell'interno del laboratorio e precisamente lungo la parete Sud. Il banco con i variometri ed il registratore fotografico sono indicati nella planimetria complessivamente con il rettangolino $b$. La fig. 4 mostra la stazione in assetto di funzionamento.

Registratore. - Tutti gli strumenti usati per le misure (4 elettrometri e 2 galvanometri) avevano bisogno della registrazione fotogra-

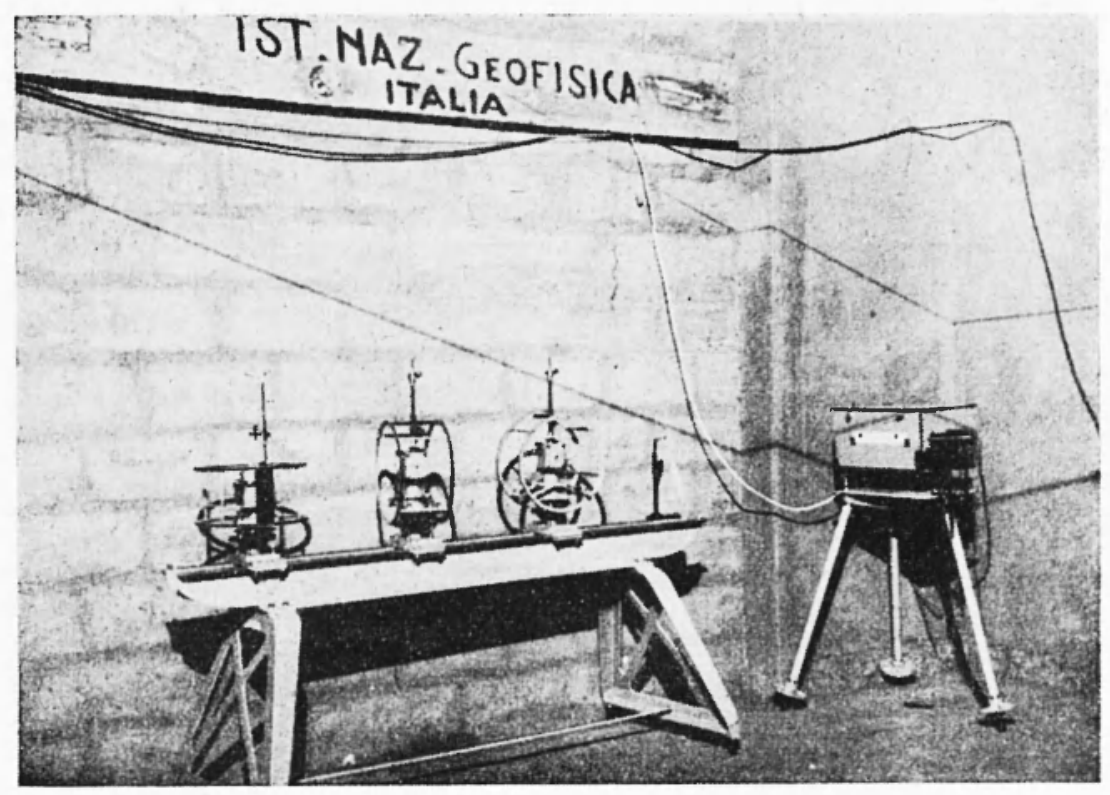

Fig. 4.

fica, ed erano posti all'interno di un grande registratore a più tracce. Questo registratore (rettangolino a della fig. 2), visibile nella fig. 1 in fase di montaggio, è mostrato nella fig. 5. Per questa fotografia sono state rialzate lateralmente le tende a tenuta di luce che coprivano normalmente il registratore, e sono visibili $i$ tre scompartimenti in cui quest'ultimo si trovava ad esser diviso. Nel primo scompartimento a destra si trovavano gli strumenti e le lampadine elettriche filiformi per la traccia luminosa; nel secondo il robusto moțore a molla ed il tamburo con la carta sensibile; l'ultimo a sinistra serviva come ca- 
mera oscura per il cambio della carta e per la carica del motore. Si vedono le linee clue dalla finestra di destra connettevano, attraverso isolatori di elettrina e bachelite, gli elettrometri ed i galvanometri agli apparati esterni. In luasso a destra si nota il pannello di controllo della sensibilità dei galvanometri, che veniva variata a mezzo di shunts inseribili con dei commutatori.

Dopo le necessarie prove, il griorno 22 febloraio furono iniziate le registrazioni regolari. 1124 felıbraio ricevemmo da Roma per via aerea, con notevole ritardo, la stazione magnetica Ruska. Il giomo

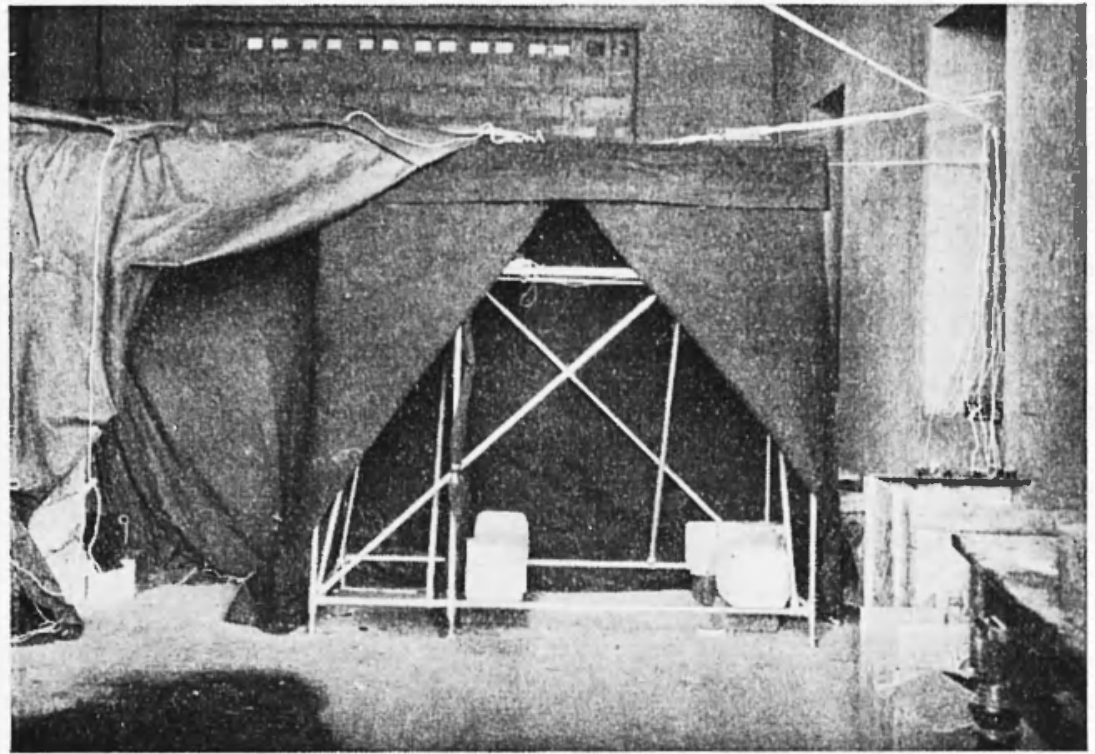

Fig. 5

dell'eclisse l'Osservatorio funzionava regolarmente e furono registrate tutte le grandezze ad eccezione della componente verticale e della declinazione del campo magnetico terrestre. Le registrazioni proseguirono poi al completo fino al 7 marzo; lo smontaggio ed il successivo imballaggio degli apparati e dei materiali fu completato entro l'8 marzo. Il 9 mattina si iniziò il viaggio di ritorno, mentre la missione astronomica aveva già lasciato Khartoum il 2 marzo. 1117 marzo eravamo di nuovo in Italia.

Esprimiamo qui la nostra gratitudine al prof. Massimo Cimino, che, con la sua attiva collaborazione, ha notevolmente contribuito allo svolgimento regolare della spedizione. 
Desideriamo inoltre ringraziare vivamente S. E. Mons. F. S. Bini, vescovo di Khartoum, e la missione cattolica di Khartoum per l'ospitalità concessa, e le rappresentanze diplomatiche e consolari italiane in Eqitto e nel Sudan per l'assistenza prestataci.

Roma - Istituto Nazionale di Geofisica - Maggio 1952.

\section{RIASSUNTO}

Gli AA. riferiscono sopra all'impianto dell'Osservatorio temporaneo installato a Khartoum in occasione dell'eclisse totale di sole del 25 febbraio 1952. Vengono poi brevemente descritte le attrezzature usate per la osservazione di alcuni fenomeni geofisici dell'ottica e dell'elettricità atmosferica e del magnetismo terrestre.

\section{SUMMARY}

The authors report concerning the setting-up of a temporary Observatory at Khartoum on the occasion of the total solar eclipse of February 25, 1952. The equipment for making observations of some geophysical phenomena (optical and electrical properties of the atmosphere and terrestrial magnetism) has been briefly described. 